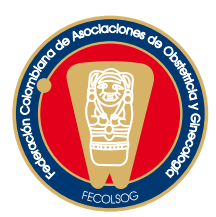

SERIE DE CASOS

\title{
AMNIOINFUSIÓN CON ÍNDIGO CARMÍN EN SOSPECHA DE RUPTURA PREMATURA DE MEMBRANAS OVULARES PRETÉRMINO. SERIE DE CASOS
}

\section{Carmine indigo amniotic infusion in patients having suspected preterm rupture of membranes}

Ana María Guevara-Zambrano, M.D. *, María Isabel Vergara-Pérez, M.D.*, Jorge Hernán Gutiérrez-Marín, M.D.**, José Enrique Sanín-Blair, M.D.***

Recibido: enero 18/07 - Revisado: mayo 24/07 - Aceptado: agosto 2/07

\section{RESUMEN}

Introducción: la detección y diagnóstico oportuno de la ruptura prematura de membranas ovulares (RPMO) en ocasiones pueden ser dudosos y requerir pruebas diagnósticas adicionales con el fin de disminuir intervenciones innecesarias, entre ellas hospitalizaciones prolongadas. La amnioinfusión con índigo carmín es una alternativa diagnóstica frecuentemente utilizada en estos casos.

Objetivo: describir la experiencia con la utilización de la amnioinfusión con índigo carmín en pacientes ingresadas a la unidad materno infantil de la Clínica Universitaria Bolivariana (CUB) con diagnóstico dudoso de RPMO.

Materiales y métodos: se realizó un estudio observacional, serie de casos, retrospectivo en pacientes ingresadas a la unidad materno infantil de la CUB con sospecha de RPMO pretérmino entre las 22 y 36 semanas, en quienes se realizó amnioinfusión con índigo carmín.

* Residente Especialización en Ginecología y Obstetricia, Facultad de Medicina, Universidad Pontificia Bolivariana, 2006.

** Gineco-Obstetra, especialista en Medicina Materno Fetal. Unidad de Medicina Materno Fetal-Clínica Universitaria Bolivariana. Profesor Universidad Pontificia Bolivariana-Universidad de Antioquia.

*** Gineco-Obstetra, especialista en Medicina Materno Fetal. Unidad de Medicina Materno Fetal-Clínica Universitaria Bolivariana. Profesor Universidad Pontificia Bolivariana-Universidad de Antioquia. Correspondencia: Calle 7 No 39-290 consultorio 1215 Medellín, Colombia. Correo electrónico: saninblair@yahoo.com
Resultados: se practicaron 41 amnioinfusiones (una paciente con embarazo gemelar), 37 fueron transamnióticas y cuatro transplacentarias. En cinco pacientes la amnioinfusión fue positiva para RPMO. Las complicaciones descritas fueron: abruptio de placenta en una paciente (amnioinfusión transplacentaria) y en tres pacientes amenaza de parto pretérmino. Cuatro pacientes en quienes la vaginoscopia fue negativa presentaron RPMO comprobada por amnioinfusión. Cinco pacientes sin RPMO presentaron oligohidramnios, y en cinco pacientes con ecografía reportada con líquido normal se confirmó RPMO.

Conclusión: la amnioinfusión con índigo de carmín es una prueba diagnóstica para considerar en el abordaje clínico de las pacientes con sospecha de RPMO.

Palabras clave: amniocentesis, oligohidramnios, indigotindisulfanato de sodio, ruptura prematura de membranas fetales.

\section{SUMMARY}

Introduction: the opportune detection and diagnosis of premature rupture of membranes (PROM) reduces unnecessary interventions such as prolonged hospitalisation; nevertheless, diagnosis can be doubtful and requires additional tests. 
Carmine indigo amnioinfusion is considered to be the gold standard in such cases.

Objective: describing carmine indigo amnioinfusion use regarding patients admitted to Clinica Universitaria Bolivariana having suspected PROM.

Materials and methods: an observational, case series and retrospective study was carried out on patients 22 and 36+6 weeks after being admitted to hospital.

Results: a total of 41 amnioinfusions were done (one patient with twin pregnancy); of them 37 were transamniotic and 4 were across the placenta. Amnioinfusion was positive for PROM in five patients. The complications found were one case of placental abruption in transplacental route and three cases of threatened preterm labour. Four of the patients in whom speculum exam proved negative presented PROM, verified by amnioinfusion. Five patients without PROM presented oligohydramnios; a diagnosis of PROM was confirmed in five patients having normal amniotic fluid ultrasound.

Conclusion: indigo carmine amnioinfusion is a diagnostic test having few complications and is useful in a clinical approach to patients having suspected PROM.

Key words: amniocentesis, oligohydramnios, indigotindisulphanate sodium, foetal membrane, premature rupture.

\section{INTRODUCCIÓN}

La ruptura prematura de membranas ovulares (RPMO) se define como la pérdida de la continuidad de las membranas corioamnióticas antes del inicio del trabajo de parto. ${ }^{1-3} \mathrm{La}$ incidencia de RPMO pretérmino fluctúa entre 2,7 y 17\% y se asocia aproximadamente a un tercio de los partos pretérmino, generando morbimortalidad fetal y morbilidad materna. ${ }^{1-4}$

El diagnóstico de RPMO, la mayoría de las veces, se realiza por una historia clínica sugestiva y hallazgos concordantes con el examen físico al observar escurrimiento de una cantidad variable de líquido amniótico (LA) por la vagina. ${ }^{2,4}$ En ocasiones clínicamente no es posible hacer el diagnóstico diferencial entre las entidades que simulan la ruptura de membranas y una verdadera RPMO, lo que lleva a utilizar procedimientos complementarios, como la inyección intraamniótica de índigo carmín, cuya detección en la vagina confirma el diagnóstico. ${ }^{1,5} \mathrm{La}$ amnioinfusión con índigo carmín es sugerida por algunos como prueba de oro para el diagnóstico de RPMO, es un procedimiento invasivo, con 1\% de complicaciones teóricas descritas que requiere ser realizada en una institución de alto nivel de complejidad. ${ }^{6,7}$

Ante la sospecha de una RPMO, las pacientes deben ser hospitalizadas, en ocasiones innecesariamente ya que no se trata de verdaderas RPMO sino de alguno de sus diagnósticos diferenciales, por lo tanto, la utilización de amnioinfusión con índigo carmín confirmaría este diagnóstico acortando estancias hospitalarias, disminuyendo costos y otras morbilidades asociadas con exámenes innecesarios y uso de antibióticos no justificados. ${ }^{8}$

El objetivo de esta publicación es describir la experiencia preliminar de la realización de amnioinfusión con índigo carmín en las pacientes con sospecha de RPMO, entidad que representa $8 \%$ de todas las hospitalizaciones en la unidad materno infantil de la Clínica Universitaria Bolivariana.

\section{MATERIALES Y MÉTODOS}

Se realizó un estudio observacional, tipo serie de casos retrospectivo, en las pacientes ingresadas con sospecha clínica de RPMO con una edad gestacional entre 22 y 36 semanas +6 días, a las cuales se les practicó amnioinfusión con índigo carmín con fines diagnósticos, en la unidad materno infantil (UMI) de la Clínica Universitaria Bolivariana entre noviembre del 2004 y mayo del 2006. Esta institución es un Hospital privado de referencia de alta complejidad de atención que atiende pacientes adscritos a la seguridad social privada en Medellín, Colombia. 
La amnioinfusión fue realizada por indicación clínica como parte del protocolo de manejo de estas pacientes y fue llevada a cabo por personal entrenado en medicina materno fetal (JESB, JHGM). La técnica incluía: bajo guía ecográfica (Volusson 730 Pro General Electric, USA), se identificó el lago mayor de líquido amniótico, se puncionó la cavidad amniótica con aguja \# 22 y se introdujo en la cavidad una mezcla de 19 cc de solución salina estéril y 1 cc de índigo carmín. $\mathrm{Al}$ terminar el procedimiento se dejó una gasa en el fondo de saco vaginal durante 3-4 horas para luego realizar la interpretación de la prueba. Se consideró como prueba positiva aquella en la que se evidenció gasa teñida de azul confirmando el diagnóstico de RPMO, y negativa en el caso contrario.

La recolección de la información se hizo mediante la evaluación de las historias clínicas de las pacientes seleccionadas a partir de la base de datos llevada por el especialista, libro de entregas de turnos en hospitalización por maternidad y base de datos de ingresos a la UMI, a quienes se les realizó amnioinfusión con índigo carmín. Las variables evaluadas fueron: edad gestacional al momento de la amnioinfusión, resultado de otras ayudas diagnósticas (vaginoscopia y ecografía), vía del procedimiento, complicaciones, estancia hospitalaria, vía del parto y resultado perinatal. El análisis de los datos se elaboró con los objetivos propuestos a través de la estadística descriptiva en el programa Epi-Info 6.04.
El estudio fue aprobado por el Instituto de Ética y Bioética de la Universidad Pontificia Bolivariana. No hubo conflicto de intereses.

\section{RESULTADOS}

Se practicaron 41 amnioinfusiones en 40 pacientes (una paciente con embarazo gemelar) ingresadas a la unidad materno infantil de la CUB con sospecha de RPMO pretérmino; 37 fueron transamnióticas y 4 transplacentarias. La edad promedio de las pacientes del grupo de estudio fue 23 años (DE: 6,07 años, rango 14-36), 16 pacientes (40\%) eran nulíparas, y entre las pacientes con embarazos previos, la mayoría (14 pacientes) eran secundigestantes. La edad gestacional de las pacientes al momento de la amnioinfusión se distribuyó así: el 23\% tenían de 24 a 27 semanas, el $49 \%$ entre 28 y 31 semanas y el 28\% 32 ó más semanas.

En cinco pacientes la amnioinfusión fue positiva para RPMO, (tabla 1). El comportamiento de la especuloscopia y de la ecografía en relación con la prueba de índigo carmín se puede observar en las tablas 2 y 3 .

En cuatro de los procedimientos se presentaron complicaciones como abruptio placentario del 10\% en una paciente que se le realizó la amnioinfusión por vía transplacentaria, y en las tres pacientes restantes, amenaza de parto pretérmino que cedió mediante útero - inhibición.

\section{Tabla 1. Características de las pacientes con RPMO confirmada por amnioinfusión}

\begin{tabular}{|c|c|c|c|}
\hline $\begin{array}{c}\text { Edad gestacional } \\
\text { a la amnioinfusión } \\
\text { (semanas) }\end{array}$ & $\begin{array}{c}\text { Edad gestacional al parto } \\
\text { (semanas) }\end{array}$ & Causa del parto & Morbilidad neonatal \\
\hline $29+4$ & $32+6$ & DCP* & Riesgo séptico \\
\hline 29 & 31 & Infección & Riesgo séptico \\
\hline 32 & $33+1$ & DCP (abruptio) & EMH* \\
\hline $34+3$ & $34+4$ & TPP* (podálica) & EMH séptico \\
\hline $26+3$
\end{tabular}

* DCP: Desproporción cefalo-pélvica; TPP: Trabajo de parto pretérmino; EMH: Enfermedad de membrana hialina. 
Tabla 2. Vaginoscopia comparada con

amnioinfusión en las pacientes con sospecha de RPMO

\begin{tabular}{|l|c|c|c}
\hline & \multicolumn{2}{|c|}{ Amnioinfusión } & \multirow{2}{*}{ Total } \\
\hline Vaginoscopia & RPMO & No RPMO & \\
\hline Positiva & 0 & 3 & 3 \\
\hline Dudosa & 1 & 3 & 4 \\
\hline Negativa & 4 & 29 & 33 \\
\hline Total & 5 & 35 & 40 \\
\hline
\end{tabular}

Tabla 3. Valoración ecográfica comparada con amnioinfusión en las pacientes con sospecha de RPMO

\begin{tabular}{|l|c|c|c}
\hline & \multicolumn{2}{|c|}{ Amnioinfusión } & \multirow{2}{*}{ Total } \\
\hline Ecografía & RPMO & No RPMO & \\
\hline Oligoamnios & 0 & 5 & 5 \\
\hline Normal & 5 & 30 & 35 \\
\hline Total & 5 & 35 & 40 \\
\hline
\end{tabular}

De las 40 pacientes evaluadas, se obtuvo información referente a la terminación del embarazo en 37 de ellas. La edad gestacional al momento del parto osciló entre 28 y 41 semanas. De las pacientes, 64,8\% (24/37) tuvieron embarazo a término; en ocho pacientes el parto fue entre la semana 34 y 36, y en cinco pacientes entre la semanas 28 y 33. El parto vaginal no instrumentado fue la vía de terminación del parto en 22 pacientes, la cesárea en 12 pacientes y fórceps en seis de ellas. La indicación principal para terminación del embarazo fue el inicio del trabajo de parto en embarazo a término (19/37).

La estancia hospitalaria de las pacientes en quienes la amnioinfusión descartó RPMO fue de 3,49 días (SD: 1,85 días, rango: 2-12 días) mientras que en aquellas con RPMO comprobada fue de 14,8 días (SD: 9,65 días, rango: 3-25 días).

\section{DISCUSIÓN}

En este estudio se describe la experiencia de la prueba de amnioinfusión con índigo carmín para esclarecer el diagnóstico de RPMO en un hospital de alta complejidad.
La consulta en urgencias de las pacientes gestantes por amniorrea es muy frecuente y constituye un reto para el médico que las evalúa. Ante la sospecha de RPMO se debe aclarar el diagnóstico oportunamente, ya que hacerlo luego de 24 horas de ocurrida aumenta la morbilidad y mortalidad perinatal. ${ }^{6}$ De igual manera, el diagnóstico erróneo o tardío de RPMO representa un factor de riesgo independiente para las complicaciones pre y posnatales. ${ }^{7}$

Realizar el diagnóstico de RPMO no es fácil y amerita siempre de un examen físico riguroso que incluya una especuloscopia, y en ocasiones exámenes complementarios como las pruebas en el líquido vaginal, que pretenden confirmar o descartar la ruptura de membranas. ${ }^{8}$ Su diagnóstico temprano se encamina a reducir las complicaciones: corioamnionitis, sepsis neonatal, hemorragia intraventricular y endometritis. $^{9}$

Cuando se descarta la ruptura de membranas, la salida de líquido por la vagina, puede ser atribuida a pérdidas inadvertidas de orina, hidrorrea gravídica y vaginosis, entre otras causas, las cuales en su mayoría no ameritan manejo hospitalario. ${ }^{10}$

Estudios previos sugieren que la ecografía, como prueba aislada, es insuficiente para el diagnóstico del RPMO,${ }^{11}$ tal como lo sugieren los hallazgos de este estudio.

Aunque la literatura reporta frecuencias de RPMO de 10\% del total de embarazos, el porcentaje de pacientes que consultan por sospecha de RPMO en urgencias es tan alto como 30\%. Lo anterior significa que solo la tercera parte de las pacientes que consultan por este motivo, realmente tienen RPMO.$^{12}$ La frecuencia observada de RPMO real en el presente estudio fue menor.

La vaginoscopia en la cual se evidencia presencia de hidrorrea es considerada por muchos como de certeza de RPMO. ${ }^{13}$ La razón para que la vaginoscopia sea positiva y sin embargo, no se compruebe la RPMO con el índigo carmín, podría ser explicada por la ruptura de solo una de las capas de las membranas (el corion) con la presencia de un amnios intacto. 
Se han descrito otras pruebas no invasivas para diagnóstico de RPMO como: la prueba de nitrazina, considerada por muchos autores como patrón de oro de RPMO $;{ }^{13,14}$ para la cual se ha descrito una sensibilidad y especificidad de 90,7\% y 77,2\%, respectivamente. ${ }^{15}$ Otras pruebas incluyen la fibronectina y el amniosure [anticuerpos monoclonales para detectar alfa microglobulina-1 placentaria], ${ }^{16-18}$ no están disponibles de rutina en nuestro medio. ${ }^{17,18}$

En cuanto a las complicaciones, la literatura reporta una frecuencia aproximada de complicaciones de $1 \% .{ }^{19}$ En el grupo estudiado, se presentó un abruptio de placenta, de acuerdo con lo descrito en la literatura, esta complicación se presenta principalmente cuando la aminioinfusión se realiza por vía transplacentaria. También se ha descrito la presentación de contracciones posteriores a la amniocentesis en el $10 \%$ de las pacientes, ${ }^{18}$ lo cual está de acuerdo con los datos descritos en este estudio. Aunque en la literatura se describe la utilización de uteroinhibición profiláctica, ${ }^{20}$ esta no se practica de rutina cuando se realizan los procedimientos en la Clínica Universitaria Bolivariana.

\section{CONCLUSIÓN}

La amnioinfusión con índigo de carmín es una alternativa que debe considerarse en el abordaje clínico de las pacientes con sospecha de RPMO.

\section{AGRADECIMIENTOS}

Los autores expresan sus agradecimientos a:

Dra. Mónica Massaro C, Coordinadora de Investigación de la Facultad de Medicina de la Universidad Pontificia Bolivariana y asesora metodológica de este estudio.

Personal de archivo clínico, Clínica Universitaria Bolivariana.

\section{REFERENCIAS}

1. Hofmeyr GJ, Gulmezoglu AM, Nikodem VC, Jager M. Amnioinfusión. Eur J Obstet Gynecol Reprod Biol 1996;64:159-65.
2. Barco MC, Noreña EA, Ocampo OJ, Salcedo ER. Manejo conservador de la ruptura prematura de membranas pretérmino. Rev Colomb Obstet Ginecol 1999;50:157-62.

3. Svigos FS, Robinson FM, Vigneswaran R. Prelabor rupture of the membranes. En: James DK, Steer PJ, Weiner CP, Gonik B, editors. High Risk Pregnancy. 3rd. ed. Philadelphia; 2005. p. 1321-33.

4. Gómez R. Rotura prematura de membranas. Boletín Perinatal. Visitado 2006 May 5. Disponible en: http:// www.cedip.cl/Boletin/BoletinRPM.htm

5. Gramellini D, Fieni S, Kaihura C, Faiola S, Vadora E. Transabdominal. antepartum amnioinfusión. Int J Gynecol Obstet 2003;83:171-8.

6. Winer A, David A, Liconte P, Aubron F, Rogez JM, Rival JM, et al. Amniocentesis and amnioinfusion during pregnancy report of four complicated cases. Eur J Obstet Gynecol Reprod Biol 2001;100:108-11.

7. Mercer BM. Preterm premature rupture of the membranes: current approaches to evaluation and management. Obstet Gynecol Clin North Am 2005;32:411-28.

8. Dugoff L, Hobbins JC, Invasive procedures to evaluate the fetus. Clin Obstet Gynecol 2002;45:1039-53.

9. Canavan TP, Simhan HN, Caritis S. An evidence-based approach to the evaluation and treatment of premature rupture of membranes: Part I. Obstet Gynecol Surv 2004;59:669-77.

10. Ramsey PS, Nuthalapaty FS, Lu G, Ramin S, Nuthalapaty ES, Ramin KD. Contemporary management of preterm premature rupture of membranes (PPROM): a survey of maternalfetal medicine providers. Am J Obstet Gynecol 2004;191:1497-502.

11. Schrimmer DB, Moore TR. Sonographic evaluation of amniotic fluid volume. Clin Obstet Gynecol 2002;45:1026-38.

12. Canavan TP, Simhan HN, Caritis S. An evidence-based approach to the evaluation and treatment of premature rupture of membranes: part II. Obstet Gynecol Surv 2004;59:678-89.

13. French JI, Mc Gregor JA. The pathobiology of premature rupture of membranes. Semin Perinatol 1996;20:344-68.

14. Ogunyemi D, Thompson W. A case controlled study of serial transabdominal amnioinfusions in the management of second trimester oligohydramnios due 
to premature rupture of membranes. Eur J of Obstet Gynecol Reprod Biol. 2002;102:167-72.

15. Norwitz ER, Park JS. Technical innovations in clinical obstetrics. Contemporary OB/GYN Technology. Visitado 2006 Abr 24. Disponible en: http:// pharmtech.mediwire.com/main/Default.aspx? $\mathrm{P}=\mathrm{C}$ ontent\&ArticleID $=181112$

16. Mc Gregor JA, Johnson S. Fig-leaf ferning and positive nitrazine testing: semen as a cause of misdiagnosis of premature rupture of membranes. Am J Obstet Gynecol 1985;151:1142-3.

17. Cousins LM, Smok DP, Lovett SM, Poeltler D. AmniSure placental alpha microglobulin-1 rapid immunoassay versus standard diagnostic methods for detection of rupture of membranes. Am J Perinatol 2005;22:317-20.

18. Hofmeyr GJ, Gulmezoglu AM, Nikodem VC, De Pager M. Amnioinfusion. Eur J Obstet Gynecol Reprod Biol 1996;64:159-65.

19. Mercer BM. Preterm premature rupture of the membranes: diagnosis and management. Clin Perinatol 2004;31:765-82.

20. Lamont RF. Recent evidence associated with the condition of preterm prelabour rupture of the membrane. Curr Opin Obstet Gynecol 2003; 15:91-9.

\section{Conflicto de intereses: ninguno declarado.}

